DOI: 10.25100/eg.v0i23.11714

Espacios y Territorios

\title{
Estrategias para potencializar el turismo de naturaleza mediado por el marketing en busca de la conservación de los bosques amazónicos
}

\section{Strategies to promote nature tourism mediated by marketing in search of conservation of the Amazonian forests}

\author{
Diana Alí García Capdevilla \\ Universidad de la Amazonia. Florencia, Colombia. \\ dia.garcia@udla.edu.co | 0000-0002-1672-6653 \\ Alexander Velásquez Valencia \\ Universidad de la Amazonia. Florencia, Colombia. \\ a.velasquez@udla.edu.co | 0000-0002-3314-097X \\ Jarrinson Alejandro Jiménez Fajardo \\ Universidad de la Amazonia. Florencia, Colombia. \\ jar.jimenez@udla.edu.co | 0000-0002-0968-2218
}

\begin{abstract}
Para citar este artículo: García Capdevilla, D., Velásquez Valencia, A., y Jiménez Fajardo, J. (2022). Estrategias para potencializar el turismo de naturaleza mediado por el marketing en busca de la conservación de los bosques amazónicos. Entorno Geográfico, (23), e20311714. https://doi.org/10.25100/eg.v0i23.11714
\end{abstract}

\section{Resumen}

En la actualidad, la deforestación es un fenómeno que ha incrementado el impacto del cambio climático. Por ello, el objeto de la investigación se enfoca en estrategias para potencializar el turismo de naturaleza mediado por el marketing en la vereda Peregrinos Caquetá Colombia en busca de la conservación de los bosques amazónicos. El tipo de investigación fue descriptiva con enfoque mixto, en la cual se empleó el diseño exploratorio, proyectivo, y experimental. La muestra correspondió a 290 personas, clasificados en turistas, líderes sociales de la comunidad y empresarios, que respondieron a los instrumentos tales como encuesta, grupo focal y entrevistas a profundidad. Los resultados se analizaron en el software Atlas ti, SPSS y se desarrolló triangulación de datos, los cuales demostraron que por medio de diferentes herramientas de mercadeo se puede posicionar al Caquetá y a Peregrinos como un lugar para realizar turismo de naturaleza, y de esta manera, promover la economía, la conservación y el desarrollo sustentable de la región.

Palabras clave: Plan de mercadeo, desarrollo, empresa.

\section{Abstract}

Currently, deforestation is a phenomenon that has increased the impact of climate change. Therefore, the object of the research focuses on strategies to enhance nature tourism 
mediated by marketing in the Peregrinos Caquetá, Colombia, in search of the conservation of the Amazonian forests. The type of research was descriptive with a mixed approach, in which the exploratory, projective and experimental design was used. The sample consisted of 290 people, classified as tourists, social leaders of the community and businessmen, who responded to instruments such as a survey, focus group and in-depth interviews. The results were analyzed in the Atlas ti software, SPSS and data triangulation was developed, which showed that by means of different marketing tools it is possible to position Caquetá and Peregrinos as a place for nature tourism, and in this way, promote the economy, conservation and sustainable development of the region.

Keywords: Marketing plan, development, company.

Recibido: 18 de junio de 2021

Aceptado: 02 de agosto de 2021

\section{Introducción}

La deforestación, es la actividad ilegal de talar, quemar y/o destruir el recurso forestal maderable; problema que durante los últimos años se ha encontrado en ascenso en Colombia, generando diferentes tipos de impactos sociales, ambientales y económicos en las comunidades rurales en Colombia, como se muestra en la Figura 1.

Fuente: Consejo Privado de Competitividad (2020).

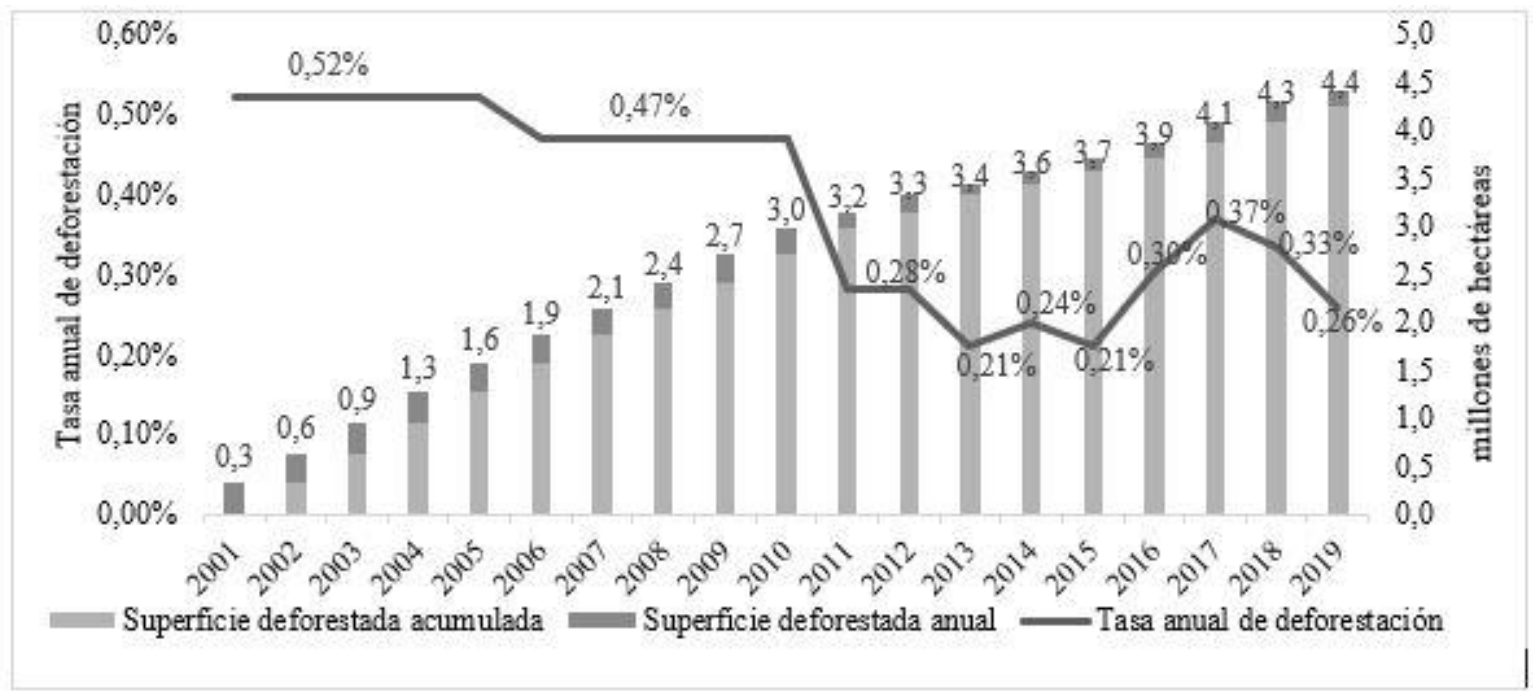

Figura 1. Histórico de deforestación en Colombia.

Caquetá, es uno de los departamentos con mayor índice de deforestación (Instituto de Hidrología, Meteorología y Estudios Ambientales - IDEAM, 2021), para el año 2020 el departamento ocupa el cuarto lugar después de Guaviare, Meta y Chocó en los niveles más altos de deforestación y destrucción masiva del bosque, a causa del uso de técnicas insostenibles, la ganadería, la contaminación y la falta de recursos económicos para garantizar una seguridad alimentaria a las comunidades rurales; estos, son los principales factores críticos determinantes de este flagelo ambiental. 
En este sentido, el turismo de naturaleza con sus diferentes actividades como caminatas ecológicas y el aviturismo (Jiménez, 2021), se convierten en una estrategia de conservación y generación de recursos económicos que satisfacen las necesidades básicas de consumo rural en concordancia con los cinco ámbitos claves, determinados como elementos esenciales de este tipo de turismo: a) crecimiento económico sustentable; b) disminución de la pobreza, aumento de empleo e inclusión social; c) mejora en el uso adecuado de los recursos, cambios en el clima y protección para el ambiente; d) fortalecimiento de la diversidad, los valores culturales y el patrimonio; e) comprensión del carácter mutuo para generar seguridad y paz (Organización Mundial del Turismo-OMT, 2017).

Pese a la gran biodiversidad presente en esta zona de la Amazonia colombiana, esta viene siendo afectada por el flagelo de la deforestación, la contaminación y las acciones destructivas bajo técnicas insostenibles, las cuales impactan los bosques, provocando con ello consecuencias en el cambio climático, tales como el incremento de la temperatura, la pauperización del aire, la extinción de especies nativas, perdiendo ecosistemas importante, lo que lleva a deteriorar el patrimonio natural primordial para el turismo de naturaleza (Estrada et al., 2019)

Además de los problemas expuestos, las especies nativas que habitan el territorio están siendo amenazadas por el incumplimiento de las leyes de protección animal y conservación, viviendo el exilio en su hábitat natural, debido a que la deforestación y la minería ilegal llevan al deterioro de la vida silvestre en el Caquetá (López et al., 2020a). Esto sucede en los 16 municipios del Caquetá, pero en los últimos años se evidencia un incremento importante en Solano denominado "La capital de la belleza", único municipio del departamento que no cuenta con vía terrestre, el cual está formado principalmente por llanuras bajas y húmedas, cubiertas de selvas vírgenes ejemplos de estas son la Serranía del Araracuara y Chiribiquete (Cataño, 2017).

En la zona rural del municipio de Solano está ubicada la vereda Peregrinos la cual se encuentra conformada por al menos 39 familias y 18.000 hectáreas de bosque amazónico que están siendo amenazadas por la deforestación y la minería ilegal, y que a través del turismo de naturaleza como estrategia sustentable busca reducir este impacto y mejorar las condiciones de vida, ingresos y empleo de la comunidad. Esta es una zona que se caracteriza por la falta de interconexión eléctrica, el mal estado de las vías de acceso y las escasas oportunidades de empleo, siendo la única fuente de su economía la agricultura transitoria con cultivos tales como yuca, plátano y maíz; cabe resaltar, que en las políticas públicas de desarrollo municipal no se encuentran contempladas actividades que generan competitividad en la vereda, por lo tanto se percibe una deficiencia de información por parte de la administración municipal (Alcaldía Municipal de Solano, 2020). Dados los problemas expuestos, esta investigación permite resolver el interrogante planteado: ¿Qué estrategias pueden potenciar el turismo de naturaleza mediado por el marketing en la vereda Peregrinos Caquetá Colombia en busca de la conservación de los bosques amazónicos? 


\section{Marco Teórico y Conceptual}

En el desarrollo de la investigación se tuvieron en cuenta los siguientes conceptos que facilitan la comprensión detallada sobre el contexto e importancia del estudio y su fundamentación desde la percepción teórica de diferentes autores. Es conveniente resaltar que el turismo, es una de las actividades económicas que busca de manera estratégica el mejoramiento de la calidad de vida a las comunidades, es por ello que según Alvarado (2019), el turismo está integrado por las actividades que realizan las personas en lugares diferentes a su entorno habitual, durante un periodo consecutivo inferior a un año, con fines de ocio, por negocios, entre otros.

De esta forma, se plantea una visión del turismo a futuro, pues hasta ahora es una de las actividades más enriquecedoras; también menciona el hecho de que diferentes tipos de factores que influyen de manera positiva y negativa en el proceso de evolución del turismo sin interesar el lugar en donde este se practique. De igual modo, Ferradás (2011) confirma el argumento de Alvarado (2019), coincidiendo en que el turismo es una actividad económica multiplicadora que integra diferentes subsectores tales como el alojamiento, restaurantes, seguros de vida, seguridad y protección consagrados en la Ley General de Turismo (Congreso de la República de Colombia, 1996).

Dado lo anterior, en el año 2012 se crea en Colombia la Política de turismo de naturaleza, en donde se interpreta que esta es una de las actividades económicas proyectadas como uno de los tipos de turismo con mayor crecimiento en el mundo; debido a las nuevas tendencias y fortalecimiento, aportando herramientas y escenarios de educación orientados hacia la conservación e interpretación de la naturaleza para tomar conciencia sobre el valor de biodiversidad en el territorio. Por lo tanto, su oferta de productos y servicios se enfoca en la naturaleza y se rige por principios de sostenibilidad; sin embargo, para su desarrollo es importante tener en cuenta que según la Organización Mundial del Turismo-OMT (2002) el turismo de naturaleza es toda modalidad caracterizada por un alto grado de apreciación hacia la naturaleza y las culturas propias de las regiones. Entre las actividades más reconocidas y practicadas en las diferentes modalidades de turismo de naturaleza, se desarrollan las siguientes:

- Ecoturismo: Observación de fauna, flora, fósiles, ecosistemas, geológica, safari fotográfico, senderismo, talleres y capacitaciones de educación ambiental, avistamiento de aves.

- Turismo de aventura: Esta actividad se enfoca en el desarrollo de caminatas, escalar cañones, ciclismo de montaña, snorkel, pesca deportiva, paracaidismo, vuelo en parapente, ala delta, globo aerostático.

- Turismo rural: Es la actividad que se enfoca en el agroturismo, visita y contextualización de pueblos indígenas, fotografía rural, vivencias rurales, 
conocimiento de medicina tradicional, capacitaciones gastronómicas, saberes artesanales.

Es así, que el departamento de Caquetá en su patrimonio natural cuenta con una variedad de 561 a 1033 especies de aves de manera específica en el municipio de Solano (Instituto de Investigación de Recursos Biológicos Alexander Von Humboldt-Instituto Humboldt, 2019), ahora bien, en esta región se encuentra ubicada la vereda Peregrinos, en donde de acuerdo con los registros del Instituto Humboldt (2019) se observan entre 561 a 720 especies en lo que se refiere al avistamiento de aves en Colombia, es por esto que en la actualidad ha declarado 54 parques nacionales y/o reservas naturales, la gran diversidad ecológica en su territorio lo hace un país atractivo para desarrollar este turismo (Fundación FAADA, 2017, p. 19); ello implica la implementación de nuevas formas de vida en los territorios donde se pretende desarrollar dicha actividad con el fin de aprovechar oportunidades de negocio relacionadas con el turismo de naturaleza, como una iniciativa para el desarrollo rural que permita la conservación del bosque amazónico en Colombia, el cual representa el 6\% de territorio latinoamericano (Organización de las Naciones Unidas para la Alimentación y la Agricultura-FAO, 2017).

Es de aclarar que, para el año 2017 en el país según cifras del IDEAM, se contaba con 59.311.350 hectáreas de bosque, representando el 52\% del territorio nacional, en donde la región Amazónica cuenta con el 66\% de participación, caso opuesto ocurre en la región Caribe donde se encuentra el 2.9\% (IDEAM; Ministerio de Ambiente y Desarrollo Sostenible, 2017).

En este contexto, los bosques protegen las cuencas hidrográficas que suministran el agua dulce a los ríos, los cuales hospedan más del $80 \%$ de la biodiversidad terrestre en la cuenca del Amazonas, en donde más de 1.300 especies de plantas forestales se utilizan para fines medicinales y culturales, consecuentemente el $12 \%$ de los bosques del mundo están designados para la conservación de la diversidad biológica (FAO, 2010). Para el 2015 las iniciativas de los bosques del mundo se basaban en la conservación del suelo y el agua, esto como resultado del suministro de aproximadamente el $40 \%$ de la energía renovable mundial en forma de combustible, es decir, energía solar, hidroeléctrica, eólica y las demás potencialidades (FAO, 2018).

Es pertinente mencionar que las áreas más representativas de bosques ecuatoriales húmedos están en la Amazonia, catalogada como el pulmón del mundo (con cerca de 8 millones de $\mathrm{km}^{2}$ ), por la gran riqueza de sus conexiones hídricas y sus bosques para la producción de agua y oxígeno, componentes esenciales de la vida y, la conservación de la mega biodiversidad que posee; según el Tratado de Cooperación Amazónica los países suramericanos de la cuenca amazónica son 8; que constituyen la más grande superficie de bosque amazónico del mundo (Rincón, 2019). 
Lo anterior, hace vital la búsqueda de la sustentabilidad, asumida como un concepto que surge para restablecer el lugar de la naturaleza en las prácticas del desarrollo, internalizando condiciones ecológicas de la producción que permitan que sobreviva la vida y se asegure un futuro para la humanidad. Este concepto engloba un proyecto social y político que apunta hacia el ordenamiento ecológico y la descentralización territorial de la producción, así como a la diversificación de los estilos de desarrollo y los modos de vida de la población que habitan el planeta; ofrece nuevos principios a los procesos de democratización de la sociedad, que inducen la participación directa de las comunidades en la apropiación y transformación de sus recursos ambientales (Mata, 2016).

\section{Contextualización del Territorio}

El Instituto Humboldt (2019), manifiesta que el departamento del Caquetá es un territorio rico en biodiversidad porque cuenta con una amplia riqueza de aves, mamíferos, anfibios, entre otros. En la actualidad el departamento se encuentra en un proceso de implementación y desarrollo de estrategias en el mercado colombiano como uno de los destinos ecoturísticos potenciales del país debido a sus bondades como la riqueza natural, la amplia variedad de especies en diferentes tipos de componentes tales como ornitología, herpetología, mastozoología, ictiología, entre otros; de acuerdo con este reporte se resaltan las maravillas naturales y las empresas turísticas más comunes y agradables de la zona, evidenciando de esta manera la generación de desarrollo territorial.

Es importante además destacar que el Caquetá es una región en donde sobresale la fauna y flora, en una amplia extensión territorial que posee ecosistemas particulares, poco explorados que se encuentran en un estado completamente natural, con potencial turístico alto en aventura y potenciales paisajes que ofrece el territorio. El Centro de Información Turística de Colombia - CITUR (2015) afirma que el departamento de Caquetá es el segundo más grande del país, con santuarios naturales representados en la flora y fauna nativa, así mismo se resalta el legado cultural en los asentamientos nativos e indígenas. Además, sobresale la presencia de parques nacionales naturales y más de 3.000 especies de vida silvestre, entre mamíferos, aves, peces, reptiles y plantas; este patrimonio natural y cultural posiciona al Caquetá como un espacio de conservación y conciencia ecológica con potencial turístico para los viajeros que llegan al país con el propósito de vivir experiencias relacionadas con el entorno natural.

Ahora bien, la cabecera municipal del municipio de Solano se encuentra localizada a 150 $\mathrm{km}$ al sureste de la capital del Caquetá - Florencia, desde donde se traslada por vía terrestre Florencia - al municipio de La Montañita (aproximadamente 20 - 30 minutos) de ahí a San Antonio de Getuchá, y desde ahí por vía fluvial por el río Orteguaza hasta Solano, ya luego de Solano rio abajo cuatro horas en deslizador hasta llegar a la comunidad de Peregrinos (Corporación para el Desarrollo Sostenible del Sur de la Amazonia-Corpoamazonia, s.f.). 


\section{Desarrollo del turismo en el Caquetá}

El Caquetá es un departamento atractivo y potencialmente turístico, principalmente por las especies que habitan el lugar, cabe resaltar que el turismo de naturaleza es una actividad de impacto económico para el departamento pues personas de otros lugares del país y de otros países están interesados en conocer el territorio caqueteño. Según España et al. (2017) “en el departamento del Caquetá, existe una gran oportunidad turística por su biodiversidad en fauna, flora y su riqueza hídrica que lo hace característico de muchos u otros lugares" (p. 10), esto se debe al bajo desarrollo económico como consecuencia de la violencia que se vivió años atrás, la insurgencia de grupos armas y los escasez de oportunidades, sin embargo, gracias al proceso de posconflicto el departamento le apuesta todo al turismo y muestra de esto es que en los últimos tres años su auge ha representado desarrollo en la región, el alza de la economía local y la educación ambiental en los ciudadanos, mejorando los aspectos socioeconómicos que potencializan en corto, mediano y largo plazo el mercado turístico, el cual permitirá que la población adquiera mayores oportunidades laborales, mejor calidad y forma de vida y desde luego la llegada a industrias nacionales que planifican la inversión y la dinamización del departamento (García et al., 2020).

\section{Método}

La investigación realizada es de enfoque mixto, que representa un conjunto de procesos sistemáticos, empíricos y críticos de investigación e implica la recolección y el análisis de datos tanto cualitativos como cuantitativos (Hernández, 2018, p. 10), el carácter fue exploratorio y proyectivo que tiene por intensión lograr el análisis y entendimiento de las estrategias establecidas en los participantes, los cuales se clasificaron en turistas, líderes sociales de la comunidad y empresarios. Por medio del método exploratorio, se realizó una revisión bibliográfica en libros, artículos científicos, tesis doctorales; analizando y extrayendo información de fuentes secundarias, terciarias y mixtas, que permitió soportar el tema de investigación. En cuanto al método proyectivo permitió el diseño de un plan estratégico de mercado a partir del resultado sobre el estudio de mercados asociado al turismo de naturaleza.

\section{Instrumento de recolección de información}

-Encuesta: Este instrumento se dirigió a 260 turistas locales, nacionales y extranjeros en el año 2019, estos fueron seleccionados de manera aleatoria simple, y la encuesta contenía 21 preguntas establecidas en un formulario mediante Google Drive y analizadas mediante un formulario de Excel (Morocho et al., 2018).

-Grupo focal: Frente a este instrumento de recolección de información, se llevaron a cabo tres sesiones de grupos focales con 6 participantes (integrantes de la comunidad) para cada uno (Flick, 2004), en donde el principal tema de discusión se enfocó en identificar la importancia del turismo de naturaleza como estrategia de conservación y reducción de la deforestación en la vereda Peregrinos; la información se analizó con el software Atlas.ti. 
-Entrevistas en profundidad: Este instrumento y técnica de recolección de información permitió obtener información de actores académicos, sector productivo y el empirismo de la comunidad frente al turismo de naturaleza (Azcuy, 2018); la información también se analizó con el software Atlas.ti. Es necesario mencionar que para la aplicación de todas las técnicas de recolección de la información los participantes dieron el respectivo consentimiento en el uso de los datos y los procesos de publicación de los resultados.

-Población: En cuanto a las cifras de visitas en el Caquetá, hubo un total de 38.000 visitantes en el año 2018 (Ministerio de Comercio, Industria y Turismo; Centro de Información Turística de Colombia-CITUR, 2018).

-Muestra: Para obtener la muestra se aplicó la técnica probabilística de sistema aleatorio simple y muestreo no probabilístico con muestreo por conveniencia; los participantes se escogieron de forma directa por la experiencia.

\section{Resultados}

De acuerdo con los instrumentos de recolección de información, se hallaron diferentes tipos de resultados de acuerdo con los grupos de interés; se inició con la revisión de la literatura especializada de la cual se analizaron las posturas de distintos autores frente a la conceptualización de las categorías de análisis. Se empleó la fórmula booleana para la revisión bibliométrica de los últimos cinco años en la base de datos Scopus, se integraron los conceptos de las categorías: turismo de naturaleza, estrategias para la sustentabilidad, conservación y bosque, por medio de la siguiente fórmula booleana: (("nature tourism"OR"sustainabilitytraining")AND("conservation"OR"regeneration")AND("forest"O R"ecosystems")), la cual, integra el turismo de naturaleza como variable independiente, conservación como variable dependiente y bosque como variable mediadora o interviniente con sus respectivos tesauros.

En razón de las variables del estudio la búsqueda bibliométrica arrojó 149 documentos científicos distribuidos en las diversas disciplinas del conocimiento en donde las ciencias ambientales tienen una participación del 30,9 \%, Agricultura 25,1\%, Ciencias Sociales 18,6\%, Ciencias de la tierra 5,2\%, Ingeniería 4,5\%. En la Figura 2 se indican los resultados referentes a la distribución según el área de investigación:

Al interrelacionar el turismo de naturaleza, conservación y bosque, se resaltan las tendencias en la dimensión de las ciencias ambientales - agricultura, ciencias sociales, mientras que el año con mayor producción académica frente a estas temáticas fue el 2018 con 35 resultados, seguido del año 2016 con 34 resultados y en tercer lugar el año 2019 con 28 resultados, en el año 2020 se encuentran 25 investigaciones publicadas. Los países que encabezan la lista de mayor producción investigativa sobre estos temas son en primer lugar, Estados Unidos con 36 resultados, seguido de Reino Unido con 17, Australia con 14 y en 
cuarto lugar un país de Sur América, Brasil con 11 investigaciones. Colombia no presenta investigaciones en los últimos cinco años sobre estos importantes temas en esta interrelación, como se evidencia en la Figura 3.

Fuente: Elaboración propia con base en resultados de Scopus (2021).

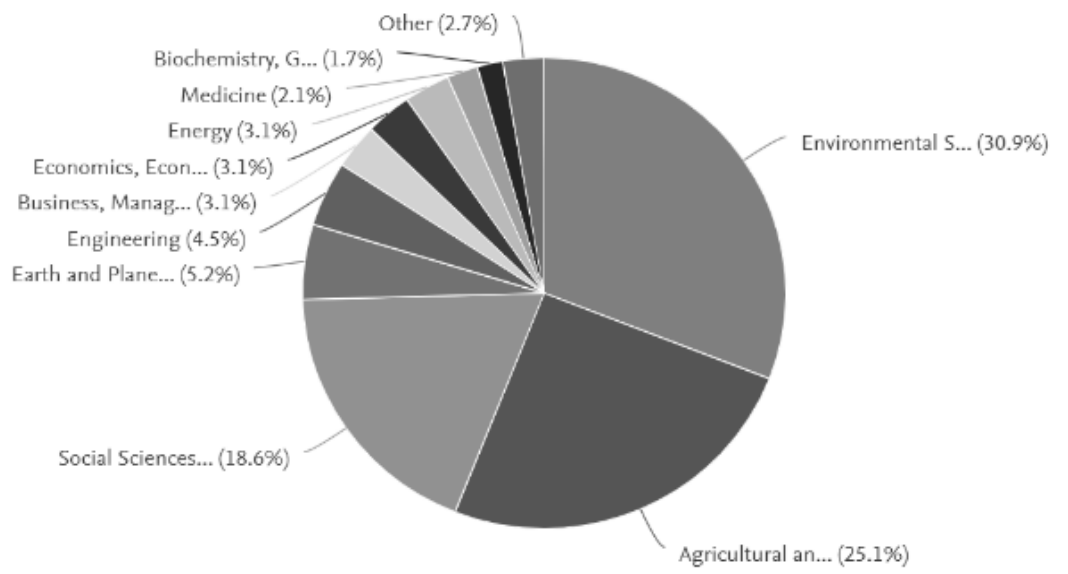

Figura 2. Distribución porcentual de documentos científicos con los términos turismo de naturaleza - conservación - bosque.

Fuente: Elaboración en Biblioshiny for bibliometrix con base en resultados de Scopus (2021).

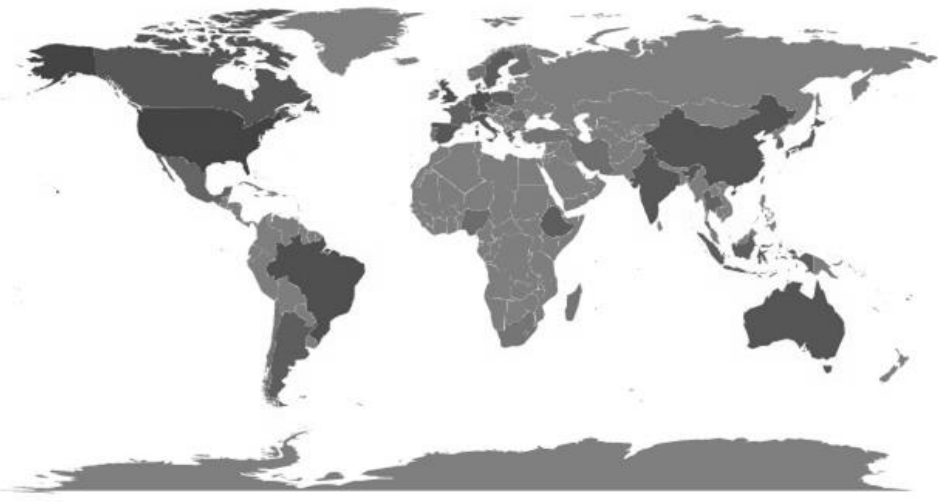

Figura 3. Producción de documentos científicos por países, con los términos turismo de naturaleza - conservación - bosque.

En la Figura 4, se observan los términos más abordados a nivel investigativo sobre esta temática de estudio: a) la biodiversidad como sostén de una gran variedad de servicios ambientales para la sociedad, los cuales se afectan por diversas causas y pierden su capacidad de recuperación (Núñez et al 2003; Andrade, 2011); b) la percepción entendida como "la forma en que cada individuo aprecia y valora su entorno, e influyen de manera importante en la toma de decisiones del ser humano sobre el ambiente que lo rodea" 
(Fernández, 2008, p. 179); c) la ecología como el estudio de las interacciones de los individuos con su ambiente físico y biológico (Sánchez y Pontes, 2010).

Fuente: Elaboración en Biblioshiny for bibliometrix base en resultados de Scopus (2021).

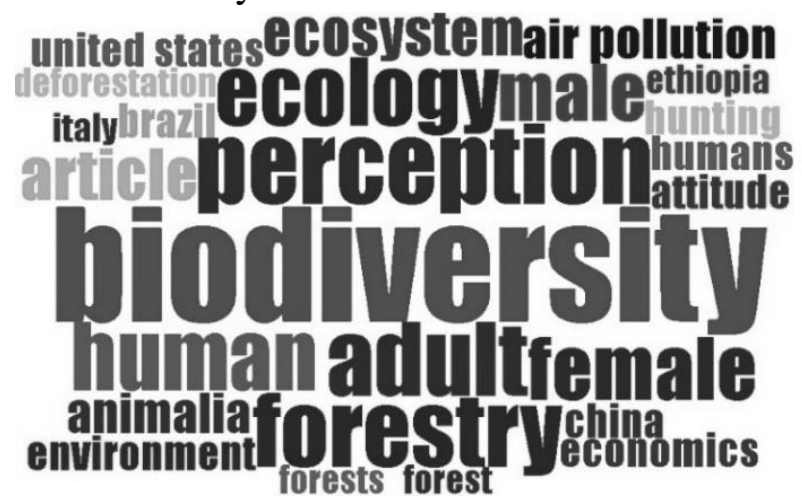

Figura 4. Términos más representativos en los documentos científicos con los términos turismo de naturaleza- conservación - bosque.

Al revisar en otras bases de datos de datos destacadas, para conocer qué se ha investigado sobre los temas relacionados con la pregunta de investigación a nivel internacional y nacional; (Science Direct y Web of Science), y en repositorios de documentos de universidades públicas y privadas, con el fin de identificar las tendencias sobre el tema que se investiga. En la revisión de dichas temáticas se elaboró la interrelación de estas categorías de análisis destacadas, para generar aportes conceptuales a las estrategias para la sustentabilidad, para la conservación del bosque en el territorio; en la Tabla 1 se muestra el resumen de las principales definiciones y características analizadas en los documentos revisados que son tendencia:

Tabla 1. Interrelación Turismo de naturaleza - conservación - bosque.

\begin{tabular}{|c|c|l|}
\hline Autor & Disciplina & \multicolumn{1}{|c|}{ Definición y Características } \\
\hline $\begin{array}{c}\text { Capellesso } \text { et al. } \\
(2020)\end{array}$ & $\begin{array}{c}\text { Ciencias } \\
\text { Ambientales }\end{array}$ & $\begin{array}{l}\text { Bosque en regeneración, es un proceso temporal para esta } \\
\text { investigación en un bosque tropical, en Brasil, concluyen que es } \\
\text { una estrategia eficaz para restaurar el suelo. }\end{array}$ \\
\hline $\begin{array}{c}\text { Giusti \& } \\
\text { (2020) }\end{array}$ & $\begin{array}{c}\text { Ciencias agrícolas } \\
\text { y biológicas }\end{array}$ & $\begin{array}{l}\text { Restauración de los bosques, esta investigación muestra la } \\
\text { urgencia en restauran ecosistemas, donde se presente la } \\
\text { interrelación de los seres humanos con la flora y fauna para la } \\
\text { sostenibilidad ecológica. }\end{array}$ \\
\hline $\begin{array}{c}\text { Tubridy (2020) } \\
\text { Ciencias sociales }\end{array}$ & $\begin{array}{l}\text { Regeneración del bosque, genera beneficios sociales, culturales } \\
\text { y ecológicos, para la adaptación climática y nuevas } \\
\text { infraestructuras 'ecológicas' y paisajísticas', con diseños } \\
\text { innovadores para mostrar la resiliencia en los espacios, que } \\
\text { pueden ser usados para el turismo de naturaleza. }\end{array}$ \\
\hline
\end{tabular}




\begin{tabular}{|c|c|l|}
\hline Autor & Disciplina & \multicolumn{1}{|c|}{ Definición y Características } \\
\hline Kull (2017) & $\begin{array}{c}\text { Ciencias de la } \\
\text { Tierra }\end{array}$ & $\begin{array}{l}\text { Bosques en transición, por la regeneración, para superar } \\
\text { flagelos como la deforestación, en países tropicales, se han } \\
\text { dedicado a trabajar la cobertura arbórea, pero sin tener en } \\
\text { cuenta que debe trabajarse con árboles nativos. }\end{array}$ \\
\hline $\begin{array}{c}\text { Hilarión y } \\
\text { Bogoya (2017) }\end{array}$ & Ingeniería & $\begin{array}{l}\text { La renaturalización, como estrategia de educación ambiental en } \\
\text { entornos escolares brinda las pautas necesarias para lograr la } \\
\text { participación de la comunidad académica garantizando la } \\
\text { apropiación del territorio, mejorando las relaciones hombre- } \\
\text { ambiente }\end{array}$ \\
\hline
\end{tabular}

Fuente: Elaboración propia con base en Scopus (2021)

Desde las disciplinas que abordan las temáticas de turismo de naturaleza, conservación y bosque, se evidencia el efecto del abandono de las tierras (intervenidas de manera antrópica), visto como un problema socioeconómico grave, pero también, puede ser una oportunidad para un nuevo desarrollo rural basado en la naturaleza con alternativas como el turismo. En este sentido, la conservación puede convertirse en esta oportunidad en muchas regiones, asegurando que los procesos naturales y las especies silvestres jueguen un papel mucho más prominente en los ecosistemas y dejando a la naturaleza cuidarse por ella misma, con una gestión menos intensiva e invasiva. De otro lado, no se evidenciaron investigaciones sobre estrategias diseñadas para contrarrestar los efectos de la deforestación y fomentar la conservación. Estas investigaciones muestran las tendencias que existen a nivel mundial y nacional, cuya premisa es la conservación del ambiente y la mitigación de la deforestación.

Por otra parte, para el desarrollo de la técnica cuantitativa se diseñó un formulario compuesto por 21 preguntas en Google Drive, las cuales se difundieron a doscientos sesenta (260) turistas a través de medios digitales como correo electrónico (Gmail y Hotmail) y el uso de redes sociales en un contexto local, nacional e internacional. Las respuestas al formulario fueron monitoreadas constantemente por el equipo de trabajo a fin de garantizar la calidad de la información. Cabe resaltar que las personas contactadas accedieron a participar y diligenciar de forma responsable el instrumento aplicado, respondiendo a todas las preguntas y siguiendo las instrucciones plateadas al inicio del formulario.

Se indagaron 179 participantes de nacionalidad colombiana (69.1\%), seguido de la población estadounidense con una cantidad de 22 personas $(8.5 \%)$, los mexicanos con 18 encuestados (6.9\%), los argentinos con 16 personas (6.2\%), los españoles con 15 personas $(5.8 \%)$, y el grupo de otras nacionalidades conformado por canadienses, brasileros y chilenos con 7 personas $(3.5 \%)$. 
Ahora bien, en la Figura 5 se identifica que el $62.5 \%$ del total de los encuestados frecuentan de 1 a 3 veces en el año, de la misma forma se observa que el $26.6 \%$ prefiere hacerlo de 4 a 6 veces al año, el $8.9 \%$ de 7 a 10 veces al año y el $1.9 \%$ les gusta hacerlo más de 10 veces al año. De lo anterior, se puede deducir en primera instancia que la demanda es positiva, la frecuencia de viajes de los encuestados incentiva a ofertar y realizar este tipo de actividades turísticas, las cuales a su vez permiten el desarrollo territorial y la conservación de los bosques en el departamento del Caquetá.

Fuente: Elaboración propia (2021).

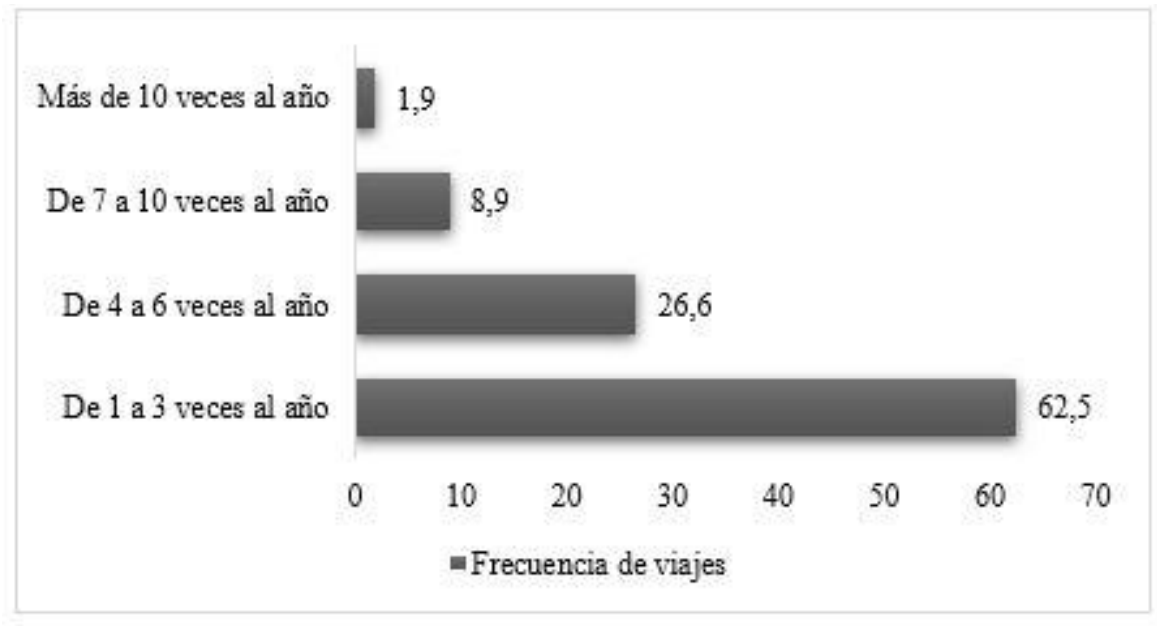

Figura 5. Frecuencia de viaje.

En cuanto a ¿cuál es la mejor temporada para realizar un viaje?, la muestra recolectada afirma que las vacaciones de fin de año es la temporada de mayor interés con un 35.9\%, seguido de los puentes festivos con 30.5\%, las vacaciones de mitad de año también obtuvieron un porcentaje significativo con un $29.6 \%$, por último, la Semana Santa con un 5 $\%$ (ver Figura 6). De lo anterior se define que es importante crear estrategias para la práctica de turismo de naturaleza en temporadas bajas del año.

En la Figura 7 se aprecia que la actividad de mayor interés para los turistas es la de aventura, con un porcentaje de $36.7 \%$, la exploración con $25.5 \%$, seguido la playa con un $23.2 \%$ y, por último, el $14.6 \%$ prefiere las actividades culturales. Es aquí, en donde se logra observar una tendencia diferente a la habitual, en donde las personas han cambiado sus preferencias de sol, playa y arena, por la aventura y la exploración, teniendo en cuenta que ambas representan el $62.2 \%$.

A continuación en la Figura 8 se muestra que de los encuestados el $41 \%$ afirma que las redes sociales son el principal medio de información para el desarrollo de actividades turísticas, guiándose por comentarios, imágenes, y puntuaciones que reflejan otros turistas en sus redes sociales, los cuales fomentan o generan críticas frente a la actividad realizada, mientras que el $25 \%$ considera oportuno recibir la información por medio de sus amigos 
que ya hayan vivido la experiencia y de acuerdo con ello toman sus decisiones, el $18 \%$ a través de agencias de turismo y finalmente están los medios de comunicación como la televisión (7\%) y el correo electrónico (9\%). Para esta pregunta los encuestados tuvieron la posibilidad de elegir más de una opción de respuesta.

Fuente: Elaboración propia (2021).

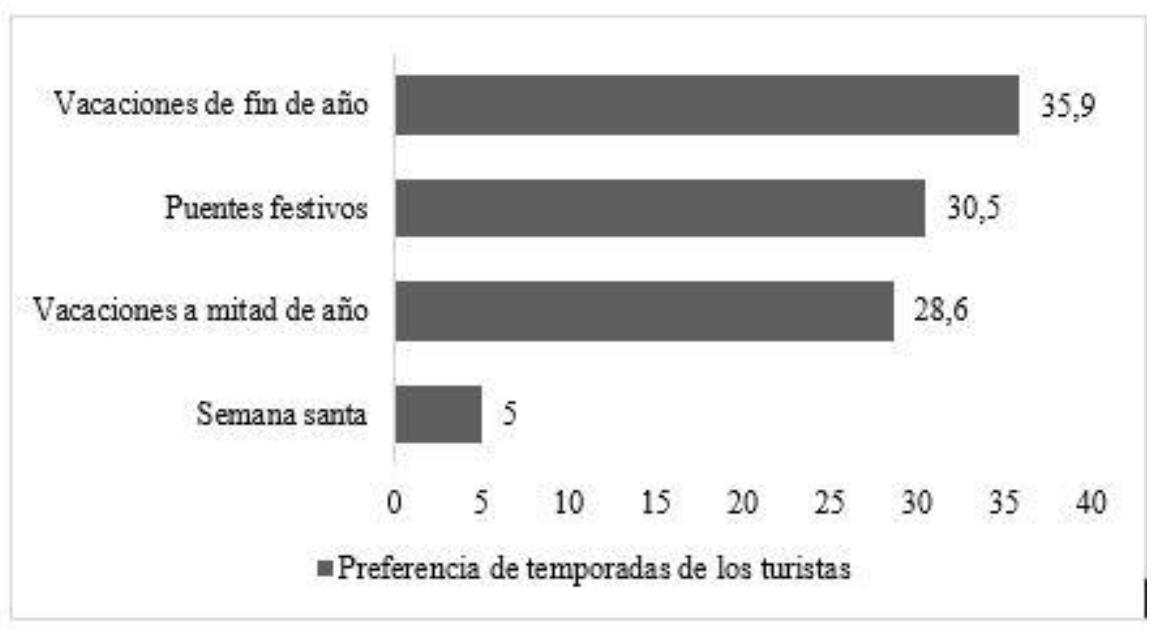

Figura 6. Preferencia de temporadas de los turistas.

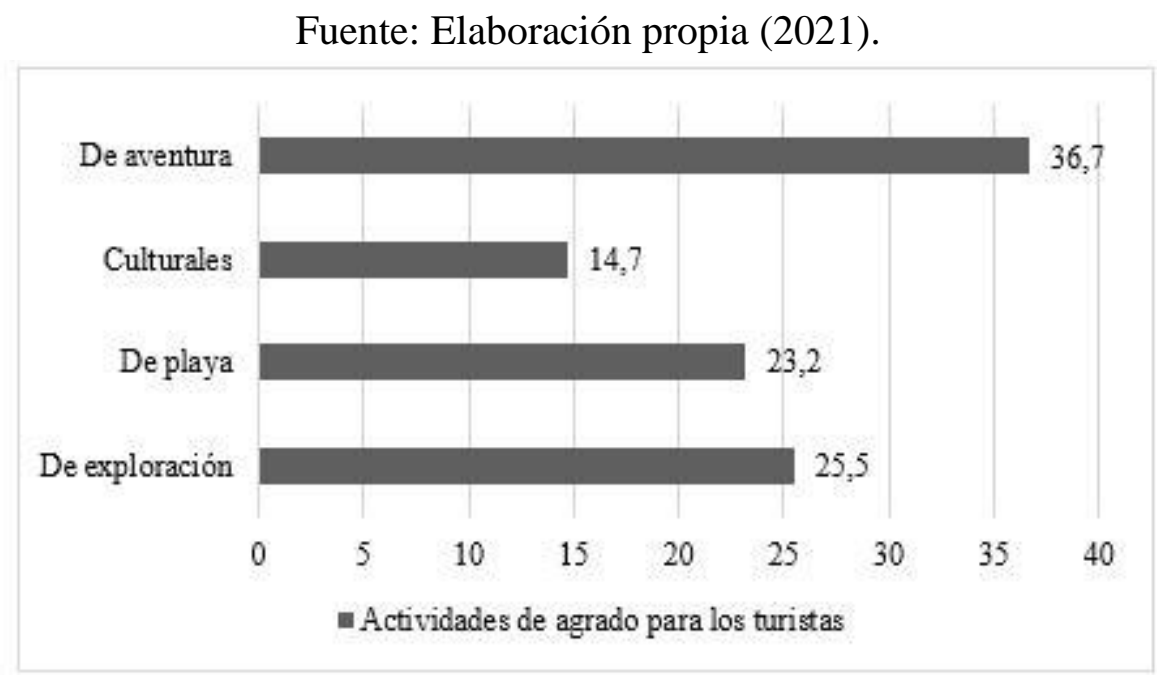

Figura 7. Actividades de mayor interés para los turistas.

Lo anterior, permitió conocer la viabilidad de potencializar el turismo de naturaleza en la comunidad de Peregrinos, como una opción que aporte a la recuperación de estas áreas que han sido afectadas por el flagelo de la deforestación, lo que han cambiado los ecosistemas causando el detrimento de la calidad ecológica y generando un paisaje de fragmentos aislados, con mosaicos de bosques en diferentes estados, los cuales por medio de la restauración o la regeneración natural pueden recuperarse, para lo cual las aves juegan un papel fundamental en la evaluación de la calidad ecológica en los bosques, como se ha 
desarrollado en el departamento de Antioquia en Colombia (Salas-Correa y ManceraRodríguez, 2020), con estrategias de marketing digital, como se corrobora en la investigación realizada en el Caquetá sobre estrategia de negocio en las MiPymes turísticas Caquetá Colombia (Aragón et al., 2019).

Fuente: Elaboración propia (2021).

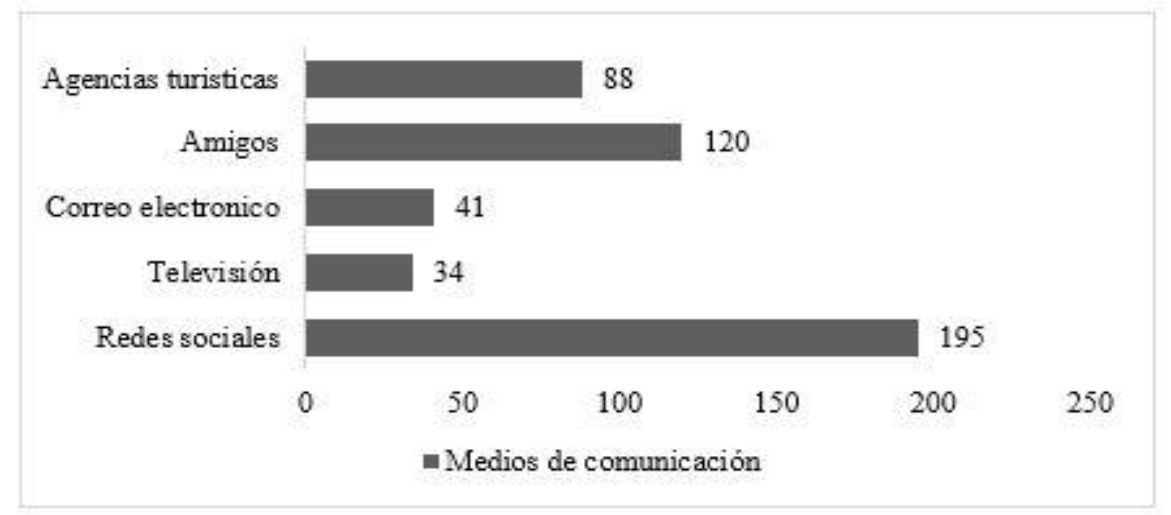

Figura 8. Medios de comunicación.

Al aplicar las entrevistas a profundidad, se logró identificar con el presidente ejecutivo de la Cámara de Comercio para el Caquetá, que el posconflicto ha permitido al sector turístico fortalecerse, y la Cámara de Comercio de Florencia se ha consolidado a través de capacitaciones y diplomados para que los operadores turísticos se preparen y se ofrezca un mejor servicio a las personas que visitan el departamento del Caquetá. Además, resalta el potencial para el desarrollo de la actividad de turismo de naturaleza, selva, fuentes hídricas y de fauna, por ello, considera se debe sectorizar para lograr ser competitivos y poder mostrar la importancia del sector turismo en el departamento. Para lo cual, se visualiza que lo importante es participar en convocatorias nacionales con el objeto de traer recursos que permitan el fortalecimiento del sector turístico, y no solo ello, si no conocimiento, el mejoramiento de habilidades y la prestación de servicios competitivos, oportunidad que se presenta de igual manera con los recursos de cooperación internacional (Entrevista. Experto $1,2020)$.

El entrevistado hace mención en que, en la actualidad se están realizando capacitaciones a los 16 municipios pertenecientes al departamento, las cuales están relacionadas con el Registro Nacional de Turismo. Por ende, indica que las 57 cámaras del país están manejando el tema del sector turismo, evidenciando perfectamente lo que se requiere en cada región, trabajando de la mano con el Fondo Nacional de Turismo (FONTUR), el Ministerio de Comercio, Industria y Turismo, y ProColombia, para obtener recursos por medio de proyectos y lograr así que el turismo dinamice la economía regional de manera sustentable, al contribuir con la conservación de la Amazonia colombiana (Entrevista. Experto 1, 2020). 
En el mismo sentido, se entrevistó al biólogo de la Universidad de Buenos Aires, dedicado a la ornitología y la observación de aves, creador de la empresa www.buenosdiasbirding.com, quien_expresa que el avistamiento de aves, como se dice en Colombia, u observación de aves como se suele decir en el resto del mundo, o birdwatching en inglés, es una afición que practican unos 78 millones de personas en el mundo, aunque no está distribuido homogéneamente, sino concentrado en ciertos países, principalmente de tradición inglesa o norte de Europa. Esta modalidad turística consiste en salir a observar las aves silvestres en libertad, en clima distendido, por el placer de estar al aire libre, y usualmente llevando un registro personal de las especies observadas. En cambio, aviturismo es la actividad de turismo asociada al avistamiento, cuando estas personas viajan a otros países y requieren de una cantidad de eslabones de una cadena que lo hace posible, esto incluye operadores, guías de observación de aves, alojamientos especializados, transportes, materiales impresos como guías de identificación, checklists, y resalta como en otros países esta actividad asociada al turismo de naturaleza ayuda a conservar los ecosistemas locales (Entrevista. Experto 2, 2020).

Se entrevistó también a un líder de la comunidad de Peregrinos, quien argumentó que el turismo de naturaleza como actividad, desde los últimos 5 años ha venido creciendo, al igual que la cantidad de turistas que llegan al territorio a realizarlo, "es claro que como negocio no es una de las actividades donde va a tener una alta demanda, pero si es una de las mejor pagadas, por ende, se cataloga como una de las actividades turísticas que más recursos genera y por lo cual he dejado la comercialización de madera para dedicarme con la comunidad al turismo de naturaleza" (Entrevista. Experto 3, 2020).

De igual manera, al desarrollar el grupo focal con la comunidad de Peregrinos, se evidenció como para los habitantes la economía se basa en el aprovechamiento del bosque, al deforestarlo para comercializar la madera; pero gracias al aporte significativo y fundamental que desarrolló el Centro de Investigación de la Biodiversidad Andino Amazónica (INBIANAM) de la Universidad de la Amazonia, con la intervención territorial mediante el desarrollo de esta investigación y el trabajo aunado con comunidades indígenas, campesinas y colonos de diferentes municipios del departamento del Caquetá, para este caso en el municipio de Solano, Corregimiento La Maná, Vereda Peregrinos, en donde se desarrolla una estrategia de implementación y mejoramiento de la calidad de vida de 39 familias mediante la conservación y el desarrollo del turismo como estrategia para el cuidado de 18.000 hectáreas de bosque.

También, con la comunidad se desarrolló una matriz DOFA (conocida también como FODA, matriz DAFO o análisis SWOT), sus siglas indican: Debilidades, Oportunidades, Fortalezas y Amenazas, como una herramienta utilizada para la formulación y evaluación de estrategias. Por medio de esta matriz se pretende identificar la situación actual de los servicios de turismo de naturaleza en la vereda Peregrinos del municipio de Solano 
Caquetá, y conocer de qué manera se pueden disminuir los riesgos a futuro y conocer su impacto en el entorno (ver Tabla 2).

- $\quad$ Factores críticos determinantes internos (Fortalezas y Debilidades).

- $\quad$ Factores críticos determinantes externos (Oportunidades y Amenazas).

Tabla 2. Matriz DOFA de la vereda Peregrinos.

\begin{tabular}{|c|c|}
\hline Fortalezas & Debilidades \\
\hline Conocimiento de las zonas turísticas de la vereda. & Dificultad de acceso a la vereda Peregrinos. \\
\hline Zona apta para brindar el servicio turístico. & Nivel mínimo de escolaridad. \\
\hline Factor diferenciador de fauna y biodiversidad. & $\begin{array}{l}\text { Falta de financiamiento en equipo tecnológico para la } \\
\text { prestación del servicio. }\end{array}$ \\
\hline $\begin{array}{l}\text { Alianzas entre la comunidad de Peregrinos y del } \\
\text { municipio de Solano. }\end{array}$ & $\begin{array}{l}\text { Ausencia de elementos necesarios para el desarrollo de la } \\
\text { práctica. }\end{array}$ \\
\hline $\begin{array}{l}\text { El servicio está directamente relacionado con el contacto } \\
\text { del medio ambiente. }\end{array}$ & Uso de las TIC. \\
\hline Amenazas & Oportunidades \\
\hline Imagen desfavorable sobre el Departamento del Caquetá. & $\begin{array}{l}\text { Generar sentido de pertenencia por los recursos naturales } \\
\text { de la región. }\end{array}$ \\
\hline $\begin{array}{l}\text { Alteración en el ecosistema por la cantidad de turistas al } \\
\text { visitar la zona, ocasionando migración en las especies. }\end{array}$ & $\begin{array}{l}\text { Generar desarrollo sostenible mediante el turismo de } \\
\text { naturaleza. }\end{array}$ \\
\hline Especies en estado de amenaza y vulnerabilidad. & $\begin{array}{l}\text { Clientes potenciales a nivel regional, nacional e } \\
\text { internacional. }\end{array}$ \\
\hline $\begin{array}{l}\text { Escases en las coberturas vegetales, ocasionando } \\
\text { migración o muerte de los animales de la zona. }\end{array}$ & $\begin{array}{l}\text { Desarrollo socioeconómico en la comunidad por medio } \\
\text { de la generación de empleo y mejoramiento de su calidad } \\
\text { de vida. }\end{array}$ \\
\hline \multirow{2}{*}{$\begin{array}{l}\text { Perdida de conectividad influenciada por las diferentes } \\
\text { actividades antrópicas. }\end{array}$} & Disminución de las actividades antrópicas. \\
\hline & Especies de distribución restringidas. \\
\hline
\end{tabular}

Fuente: Elaboración propia (2021)

Con base en lo anterior, para la promoción del producto de turismo de naturaleza se propone realizar las estrategias descritas en el siguiente punto de este artículo bajo el marco de los resultados obtenidos con el desarrollo de las técnicas aplicadas; observando la importancia de los medios digitales como una de las estrategias más viables para ofertar la actividad turística enfocada hacia el turismo de naturaleza y sus subproductos.

\section{Discusión}

El turismo de aventura, como actividad económica ha crecido en los últimos años en Colombia debido a su potencial y a la amplia biodiversidad con la que cuenta. Según López et al. (2020b) mediante su investigación muestran la importancia que tienen los programas de conservación y protección de los ecosistemas en promover iniciativas responsables con el ambiente, específicamente en la región Amazónica, en donde este hace mención que el turismo de naturaleza es una de las estrategias de planificación e integración para el mejoramiento de la calidad de vida en las comunidades rurales, brindando de esta manera la 
dinamización territorial bajo un plan de acción enfocado a la reducción del impacto ambiental en dicha región.

Afirmando el objeto de la investigación, según López et al. (2019), el turismo de naturaleza es una estrategia de conservación de los ecosistemas, y es una estrategia sustentable para mitigar la deforestación en la Amazonia colombiana, siempre y cuando esta actividad se desarrolle bajo el marco de técnicas de buenas prácticas de manejo ambiental, para un menor impacto en el territorio que se desarrolla esta práctica (Ministerio de Comercio, Industria y Turismo, 2017).

De manera que, se propone como respuesta al interrogante planteado y de acuerdo con los resultados obtenidos, un conjunto de estrategias para potencializar el turismo de naturaleza en la Vereda Peregrinos Caquetá Colombia, en busca de la conservación de los bosques, por medio de la formación a la comunidad para desarrollar actividades turísticas que se oferten por medio del marketing que permita generar propuestas de generación de valor (en torno a la conservación de los ecosistemas), para transmitir de manera objetiva esas ventajas que se pueden aportar a los turistas (Aragón et al., 2019; Basto et al., 2019; García et al., 2020; García et al., 2021).

En síntesis, las estrategias de marketing para potencializar el turismo de naturaleza en esta propuesta se consideran el pilar de la conciencia para el fomento de una cultura ambiental responsable y ética, en aras de promover la conservación de los bosques y, por ende, la disminución de la deforestación. Es por ello que esta estrategia se enfoca hacia el uso y manejo de las Tecnologías de la Información y las Comunicaciones (TIC), para desarrollar publicidad mediante la creación de páginas web, el diseño de páginas en las redes sociales asociadas a Facebook e Instagram, los videomarketing y la participación en eventos nacionales e internacionales de turismo.

\section{Estrategia de Promoción}

Para la promoción del producto se propone realizar las siguientes estrategias bajo el marco de los resultados obtenidos mediante el desarrollo de la encuesta aplicada al público objetivo. Los medios electrónicos son el factor determinante crítico más viables para ofertar la actividad turística enfocada hacia el aviturismo, de ahí que esta estrategia se direcciona hacia el uso y manejo de las TIC y la publicidad mediante el uso de las redes sociales (García et al., 2021).

- Creación de página web: Al ser una propuesta encaminada a ofertarse a nivel nacional e internacional, se propone la creación de una plataforma con información que permita al potencial cliente reconocer los atractivos del lugar y planear su viaje. Uno de los beneficios que se logran obtener por medio de esta plataforma es un mayor alcance, menor inversión en publicidad, brindar una 
retrospectiva diferente de la vereda Peregrinos y así mismo el incremento de las ventas.

- Diseño de páginas en las redes sociales asociadas a Facebook e Instagram: Es importante considerar el alto impacto de estas redes, que ayudan a la rápida propagación de información a través de las comunidades virtuales que no representen un alto costo pero con gran alcance de credibilidad en el segmento, a través de ellas se puede mostrar publicaciones de vivencias de los visitantes que desarrollan turismo de naturaleza, videos del lugar, las aves y transmitir la emoción de realizar estas prácticas; este tipo de contenido permite conectar con el público y despertar su interés. Cabe resaltar, que uno de los atributos más importantes de estas plataformas es que se puede pagar pautas publicitarias para llegar a más personas y consolidar clientes.

- Videomarketing: Se plantea como una estrategia para atraer clientes que consiste en crear contenido en vivo, el cual muestra cada una de las aventuras que se pueden apreciar en el lugar, esto ayuda a incrementar la cantidad de clientes interesados $\mathrm{y}$ a tener un mayor reconocimiento nivel regional, nacional $\mathrm{e}$ internacional.

- Participar en eventos referentes al turismo: Hacer presencia en las distintas ferias a nivel nacional e internacional con afluencia de gente a través de stands, conferencias, presentaciones audiovisuales y demás herramientas visuales.

\section{Conclusiones}

De acuerdo con la investigación realizada, se concluye que la deforestación es un problema grave a nivel mundial, y de manera específica, se evidencia en la vereda Peregrinos. Este flagelo trae consigo la necesidad de crear estrategias sustentables para el bosque andino y basal; por lo tanto, se presenta el turismo de naturaleza mediado por el marketing como la mejor alternativa para desempeñar un rol destacado en el desarrollo de la comunidad que les permita dejar de comercializar la madera y desarrollar actividades más amigables con el ambiente en busca de recuperar de los ecosistemas.

De igual manera, se evidencia que los pobladores identifican ampliamente el contexto general de la deforestación como problema ambiental en la zona que habitan, y han asumido un rol importante para hacer parte de iniciativas a cargo del Estado y las Juntas de acción comunal, con el objetivo claro de generar alternativas productivas en el marco del turismo de naturaleza y disminuir así este flagelo que genera grandes impactos negativos para el entorno. 
En el mismo sentido, se evidencia que la vereda Peregrinos tiene una gran oportunidad para el desarrollo de las actividades de turismo de naturaleza por su ubicación geográfica y su patrimonio natural caracterizado por la variedad en espacios para observación de fauna y flora; es así como, el lugar puede posicionarse frente a otros sitios turísticos del departamento y del país.

Con los resultados obtenidos durante la aplicación de los instrumentos de recolección de la información, se puede mencionar que las TIC son un factor crítico determinante para el posicionamiento y la generación del Good Will bajo el marco del turismo en la vereda Peregrinos. Así mismo, se evidencia que hay una gran demanda en el mercado nacional e internacional, puesto que, según la investigación las personas mayores y profesionales científicos son los más interesados por el avistamiento de aves a nivel mundial. Esta práctica en los últimos años ha tenido auge como una de las actividades más interesantes a desarrollar, ventaja que otorga veracidad para su implementación.

Del mismo modo, para el desarrollo turístico de esta actividad es importante precisar en conservar y proteger, por tal motivo es necesario implementar medidas de cuidado y concientización tanto para la comunidad residente del lugar como para los turistas. Para culminar, es clara la viabilidad de implementar el turismo de naturaleza en la vereda Peregrinos, debido al desarrollo social, económico y cultural que trae consigo, al igual que el fortalecimiento de estrategias para proteger y conservar el territorio.

\section{Agradecimientos}

Agradecemos al proyecto Colombia BIO-Expedición Caquetá BIO "Fortalecimiento de la gestión integral de la biodiversidad y los servicios ecosistémicos para el establecimiento de herramientas que contribuyan a su conservación en áreas de posacuerdo del departamento del Caquetá", BPIN 2018000100003.

\section{Referencias bibliográficas}

Alcaldía Municipal de Solano (2020). Plan de desarrollo territorial del Municipio de Solano, Caquetá (2020). https://bit.ly/3leACqE

Alvarado, L. (2019). Oferta turística y barreras de entrada del mercado de avistamiento de aves en operadores turísticos, distrito de Tarapoto, 2018 [tesis de maestría, Universidad César Vallejo]. Repositorio Institucional. https://bit.ly/3HZ5KEr

Andrade, M. G. (2011). Estado del conocimiento de la biodiversidad en Colombia y sus amenazas. Consideraciones para fortalecer la interacción ciencia-política. Revista de la Academia Colombiana de Ciencias Exactas, Físicas y Naturales, 35(137), 491507. 
Aragón, J. A., Corredor, V. A. y García, D. A. (2019). Estrategia de negocio en las MiPymes turísticas, Caquetá Colombia. Revista Facultad de Ciencias Contables, Económicas y Administrativas FACCEA, 9(2), 133-141. https://bit.ly/3rgiPDi

Azcuy, V. R. (2016). La entrevista en el estudio teológico de la espiritualidad. Presupuestos epistemológicos, investigación cualitativa y aportes de una técnica. Revista Teología, 53(121),73-98. https://bit.ly/3CU18hv

Basto, N. A., García, D. A. y Vargas, H. F. (2019). Estrategia de marketing digital mediante la realidad virtual para potencializar el ecoturismo en la vereda El Manantial Municipio de Florencia-Caquetá Colombia. Revista Entorno Geográfico, (18), 128-147. https://doi.org/10.25100/eg.v0i18.8743

Cataño, J. M. (23 de octubre de 2017). Solano, Caquetá, una belleza abandonada en el corazón de la selva. Noticias a la vanguardia de la información. https://bit.ly/3cRuTCN

Capellesso, E. S., Cequinel, A., Marques, R. \& Mendes, M. C. (2020). Temporal and environmental correlates of carbon stocks in a regenerating tropical forest. Applied Vegetation Science, 23(3), 353-362. https://doi.org/10.1111/avsc.12487

Centro de Información Turística de Colombia. (2015). Estadísticas departamentales Nacionales. https://bit.ly/3xwcaWA

Congreso de la República de Colombia (1996). Ley 300 de 1996. Por la cual se expide la Ley General de Turismo y se dictan otras disposiciones. https://bit.ly/3nU3CFX

Corporación para el Desarrollo Sostenible del Sur de la Amazonia-Corpoamazonia (s.f.). Municipio de Solano. https://bit.ly/2ZtX7Ai

España, H. E., Rodríguez, P. Y., Álvarez, M. A. y Olaya, N. (2017). Actividades productivas y competitivas para un clúster turístico en Florencia-Caquetá. Revista Facultad de Ciencias Contables, Económicas y Administrativas FACCEA, 7(1), 5-11. https://bit.ly/30Zg7HP

Estrada, G. E., Guzmán, M. A. y Parra, J. P. (2019). Estado actual de la fauna silvestre posdecomiso en el departamento del Caquetá-Colombia. CES Medicina Veterinaria y Zootecnia, 14(3), 64-85. https://doi.org/10.21615/cesmvz.14.3.6

Fernández, Y. (2008). ¿Por qué estudiar las percepciones ambientales? Espiral: Estudios sobre Estado y Sociedad, 15(43), 179-202. https://bit.ly/3lgNWed 
Ferradás, S. (2011). Geografía económico-turística, medioambiental y urbana: usos sostenibles en municipios turísticos. Revista Entorno Geográfico, (7-8), 8-22. https://bit.ly/3xuDSTE

Flick, U. (2004). Introducción a la Investigación Cualitativa. Ediciones Morata.

Fundación FAADA (2017). Turismo Responsable con los Animales. https://bit.ly/3nYsD2X

García, D. A., Vargas, H. F. y Restrepo, J. J. (2020). El turismo de naturaleza: educación ambiental y beneficios tributarios para el desarrollo del Caquetá. Revista Aglala, 11(1). 107 - 132. https://bit.ly/3cWz1S0

García, D. A., López, L. y Millán, E. E. (2021). Contexto y condiciones del eco marketing para promocionar el turismo de naturaleza. Revista EIA, 18(35), 1-20. https://doi.org/10.24050/reia.v18i35.1419

Giusti, M. \& Samuelsson, K. (2020). The regenerative compatibility: A synergy between healthy ecosystems, environmental attitudes, and restorative experiences. Plos One, 15(1), e0227311. https://doi.org/10.1371/journal.pone.0227311

Hernández, R. (2018). Metodología de la investigación: las rutas cuantitativa, cualitativa y mixta. McGraw Hill. https://bit.ly/3D1msPE

Hilarión, Y. y Bogoya, E. (2017). Apropiación de espacios escolares a través de la Renaturalización como estrategia de educación ambiental, en el marco del programa Servicio Social Ambiental (SSA) del Jardín Botánico de Bogotá José Celestino Mutis [tesis de pregrado, Universidad Distrital Francisco José de Caldas]. Repositorio institucional. https://bit.ly/3rf7enX

Instituto de Investigación de Recursos Biológicos Alexander Von Humboldt (2019). Cauca, Nariño y Antioquia, los departamentos con más aves en Colombia. https://bit.ly/3rglNrq

Instituto de Hidrología, Meteorología y Estudios Ambientales; Ministerio de Ambiente y Desarrollo Sostenible. (2017). Resultados Monitoreo de la Deforestación 2017. https://bit.ly/3104LCV

Instituto de Hidrología, Meteorología y Estudios Ambientales. (enero-marzo del 2021). Boletín de Detección Temprana de Deforestación (DTD). https://bit.ly/3D0rooa

Jiménez, J. A. (2021). Economía de Selva en la Amazonia Suramericana. Interconectando Saberes, 11(6), 73-85. https://doi.org/10.25009/is.v0i11.2670 
Kull, C. (2017). Forest transitions: a new conceptual scheme. Geographica Helvetica, 72(4), 465-474. https://doi.org/10.5194/gh-72-465-2017

López, L., García, D.A. y Lozano, R. (2019). Turismo de Naturaleza como estrategia sostenible para mitigar la deforestación en la Amazonia Colombiana. Ponencia presentada en Diálogo de saberes: "El rol de la educación y la participación en el contexto de la deforestación de La amazonia colombiana”. Minambiente. Florencia Caquetá.

López, L., García, D. A., Balanta, V. J. y Agundez, A. (2020). La investigación en educación ambiental y deforestación: aportes para la formación ambiental comunitaria para evitar la degradación de los bosques. Revista Perspectivas Educativas, 10(1), 93131. https://bit.ly/3xynBgO

López, L., Balanta, V. J. y García, D. A. (2020b). Asociaciones del territorio para la sustentabilidad del ecoturismo. En L. López de Parra (Ed.), Territorio y Ecoturismo: Aspectos Sociodemográficos y expectativas de inserción laboral en el Pos-acuerdo, Florencia Caquetá (pp. 57-104). Editorial Universidad de la Amazonia.

Mata, E. (2016). Turismo de naturaleza y sustentabilidad ;Un desafío para la práctica turística? [tesis de pregrado, Universidad Autónoma del Estado de México]. Repositorio institucional. https://bit.ly/3E3iO9i

Ministerio de Comercio, Industria y Turismo. (2017). Guía de Buenas Prácticas para la Actividad de Aviturismo en Colombia. https://bit.ly/3oZFoti

Ministerio de Comercio, Industria y Turismo; Centro de Información Turística de Colombia. (2018). Estadísticas en departamentos de Colombia. https://bit.ly/3xx8HHG

Morocho, Á. A., Vinueza, S. X., Andrade, C. F. y Quevedo, M. R. (2018). Evaluación del uso de técnicas aplicadas en la investigación. Revista Científica de Investigación actualización del mundo de las Ciencias, 2(3), 722-738. https://bit.ly/3nU847D

Núñez, I., González, E. y Barahona, A. (2003). La biodiversidad: historia y contexto de un concepto. Interciencia, 28(7), 387-393. https://bit.ly/3xvXFlL

Organización de las Naciones Unidas para la Alimentación y la Agricultura. (2010). Global Forest Resources Assessment. https://bit.ly/3E76wwY 
Organización de las Naciones Unidas para la Alimentación y la Agricultura. (julio del 2017). El Estado de los bosques y el sector forestal en la región. https://bit.ly/3rliZcR

Organización de las Naciones Unidas para la Alimentación y la Agricultura. (2018). El Estado de los bosques del mundo. https://bit.ly/315cAYb

Organización Mundial del Turismo. (2002). Cumbre mundial del Ecoturismo: informe final. Educación Para El Desarrollo Sostenible. https://bit.ly/3d1pGYR

Organización de las Naciones Unidas para la Educación, la Ciencia y la Cultura. (2017). Educación Para El Desarrollo Sostenible. https://bit.ly/2ZxEueU

Rincón, H. H. (2019). Amazonia Colombiana: Geografía, Ecología y Ambiente. Editorial Universidad de la Amazonia. https://bit.ly/3oZH5XG

Salas-Correa, A. D. y Mancera-Rodríguez, N. J. (2020). Aves como indicadoras ecológicas de etapas sucesionales en un bosque secundario, Antioquia, Colombia. Revista de Biología Tropical, 68(1), 23-39. DOI 10.15517/RBT.V68I1.34913

Sánchez, F. J. y Pontes, A. (2010). La comprensión de conceptos de ecología y sus implicaciones para la educación ambiental. Revista Eureka sobre Enseñanza y Divulgación de las Ciencias, 7, 270-285. https://bit.ly/3I2wIef

Tubridy, D. (2020). Green climate change adaptation and the politics of designing ecological infrastructures. Geoforum, 113, 133-145. https://doi.org/10.1016/j.geoforum.2020.04.020 